\title{
Seltene neurologische Erkrankungen
}

Diese Ausgabe der Nervenheilkunde widmet sich seltenen Erkrankungen in der Neurologie, also definitionsgemäß Erkrankungen des Nervensystems, die $\leq 1 / 2000$ Menschen betreffen. Die Einteilung, das Herangehen an ein sich im klinischen Kontext stellendes Problem als „selten“ oder „häufig" ist unnatürlich. Symptome, klinische Zeichen, Konstellationen haben für betroffene Patienten keine spezielle Qualität oder Bedeutung durch die Zuschreibung als selten oder häufig, sondern in sich durch ihren belastenden und einschränkenden Charakter. Ob nun das Zittern der Arme im Rahmen eines (häufigen) essenziellen Tremors oder als ein Zeichen eines (seltenen) orthostatischen Tremor-Syndroms als häufig oder selten klassifiziert wird, ist für die Alltagseinschränkung, das Erleben eines Patienten weniger von Bedeutung als das Verständnis des Problems und natürlich die Einleitung einer lindernden Behandlung.

Auch werden kategoriale oder dimensionale Gruppierungen von Erkrankungen bzw. Störungen nicht in erster Linie nach der Prävalenz vorgenommen.

Bereits im - weltweit - ersten Lehrbuch der Neurologie, dem wegbereitenden Lehrbuch der Nerven-Krankheiten des Menschen von Moritz Heinrich Romberg, das 1840 erstmalig erschien (1), werden, einer heute nicht mehr ganz so üblichen Einteilung neurologischer Erkrankungen in die „Sensibilitätsneurosen“ und die „Motilitätsneurosen" folgend, selbstverständlich Häufiges, wie die Zeichen eines Schlaganfalls oder einer Migräne, und Seltenes, wie die Manifestation einer "Hemiatrophia faciei“ (Rombergsche Krankheit) oder einer Obturatorius-Neuralgie (Romberg-Syndrom) behandelt. Die Gliederung heutiger Lehrbücher unterscheidet sich markant von der des Rombergschen Werkes, vor allem wegen zahlloser Fortschritte in Hinblick auf neuroanatomische und pathophysiologische Erkenntnisse, nicht in erster Linie wegen sich wandelnder Häufigkeiten. Dabei wäre ein Vergleich zwischen damaliger und heutiger Prävalenz definierter Erkrankun- gen bzw. Syndrome, wie z. B. der Syphilis, der Bleivergiftung oder psychogener Lähmungen, durchaus von Interesse. Prävalenzerhebungen und -betrachtungen können wichtige Beiträge zum Verständnis von Erkrankungen liefern, stellen jedoch, wie dargelegt, keine nosologisch bedeutsame Kategorisierungsgrundlage dar.

Warum könnte es dennoch sinnvoll sein, den Begriff „selten“ zu verwenden und dem Seltenen in dieser Ausgabe der Nervenheilkunde besonderen Raum zu geben? Nicht aus Vermessenheit, in wenigen Beiträgen das Spektrum der bekannten seltenen neurologischen Erkrankungen, derzeit etwa 1000, abzuhandeln oder überhaupt nur vorzustellen. Vielmehr aus medizin-politischen, strukturellen und anschaulichen Gründen.

Dass die Beschäftigung mit Seltenem die Neurologie kennzeichnet und maßgeblich prägt, ist offensichtlich und war bereits zu Rombergs Zeiten klar. Ebenso klar ist, dass diese Beschäftigung naturgemäß schwierig sein kann, Spürsinn und Expertise erfordert und - Zeit. Sich klinisch ungewöhnlich präsentierende, möglicherweise seltsame Probleme auszuloten, zu verstehen und zuzuordnen, kann dauern. So sinnvoll die Bestimmung und das Bestreben einer Verkürzung einer „Door-toneedle-time" zur Verbesserung des Therapieerfolges einer hoch-effektiven Schlaganfallbehandlung sind, so sinnentleert ist das Bemessen der Güte oder Nützlichkeit von Erkenntnis- und Verstehensprozessen komplexer klinischer Szenarien anhand von Zeiteinheiten - oder mittels Instrumenten einer mechanistischen Prozessund Erlösoptimierung. In den derzeitigen vorrangig betriebs- (nicht volks-) wirtschaftlich determinierten Gesundheitsstrukturen lassen sich mit dem Instrument der DRGs die für das Verstehen und die angemessene Behandlung vieler Erkrankungen notwendigen Denk- und Handlungsprozesse, also Kernelemente der ärztlichen Kunst, nicht abbilden. Anders ausgedrückt: Wer ärztliche Kunst, Kraft und Zeit im System nicht günstig abgebildeten

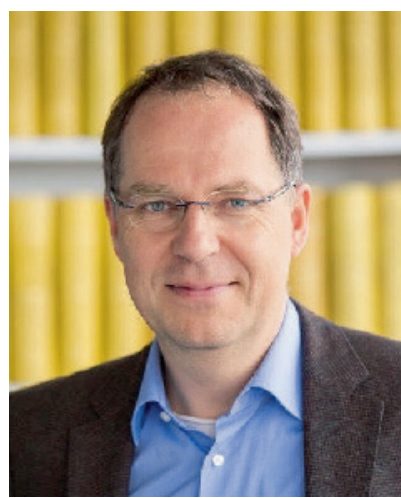

Prof. Dr. med. Alexander Münchau, Leitung, Zentrum für seltene Erkrankungen, Lübeck 
Vorgängen und Ereignissen widmet, schadet dem Betrieb. Aus betriebswirtschaftlicher Logik müssen diese Vorgänge sanktioniert werden, z. B. durch Schließung zeitintensiver Spezialambulanzen. Die flächendeckenden, für ärztlichen Freiraum, Expertiseentwicklung, Patientenbetreuung und Nachwuchsförderung disruptiven Konsequenzen überwiegend betriebswirtschaftlicher Regeln sind gut belegt und mittlerweile allgemein bekannt (2-4).

Nun sind Patienten mit „schwierigen“ Erkrankungen aber weiter da und klopfen an. Und es gibt viele, die sich weiterhin mit diesen Patienten beschäftigen. Weniger aus intrinsisch medizinischer als vielmehr struktur-politischer Notwendigkeit ist in diesem Zusammenhang der Terminus ,seltene Erkrankung“ zu einem Konstrukt und Siegel geworden, das betroffenen Patienten mit „schwierigen“, zeitaufwendigen Erkrankungen, die aus betriebswirtschaftlichen Erwägungen des Raums der Gesundheitswirtschaft verwiesen wurden, nun wieder Türen öffnet, $z$. B. in neu gegründeten Zentren für Seltene Erkrankungen (ZSEs). Das durch die Bundesregierung berufene Nationale Aktionsbündnis für Menschen mit Seltenen Erkrankungen (NAM$\mathrm{SE}$ ), die Allianz Chronischer Seltener Erkrankungen (ACHSE) und weitere Interessengruppen und Patientenorganisationen haben dies maßgeblich befördert und vom System Benachteiligten eine kräftige Stimme gegeben. Vielerorts sind ZSEs entstanden, in deren innerer Struktur A-Zentren als krankheitsübergreifende Referenzzentren, B-Zentren als Fachzentren, die auf die Betreuung einer Gruppe seltener Erkrankungen spezialisiert sind und C-Zentren als niedergelassene Schwerpunktpraxen zur wohnortnahe Versorgung von Menschen mit seltenen Erkrankungen, konzipiert sind (5). Bislang wurden in Deutschland 28 ZSEs gegründet. Kürzlich wurden durch die EU darüber hinaus 26 Europäische Referenznetzwerke (European Reference Networks; ERN) genehmigt, die existierende Expertise-Zentren europaweit verbinden. Hierzu gehören auch das ERN on Rare Neurological Diseases (ERN-RND), das ERN on Neuromuscular Diseases (EURO-NMD) und das ERN on Epilepsies (EpiCARE). Ziele dieser Netzwerke sind eine standardisierte und interdisziplinäre $\mathrm{Pa}$ - tientenbetreuung, ein enger Verbund von klinischer Behandlung und Forschung sowie internationale Patientenregister (6).

Die Gründung von ZSEs, ERNs und weiterer Netzwerken ist ein nicht zu unterschätzendes Signal, löst die Probleme der Patienten mit seltenen Erkrankungen aber solange (noch) nicht, bis diese Zentren in ihrer auf die Bedürfnisse zugeschnittenen Ausstattung mit Personal, also z. B. Ärzten, Studienkoordinatoren, medizinischen Fachangestellten und Räumen angemessen finanziert werden. Hierzu gibt es erste Modelle, wie das vom Innovationsausschuss geförderte Projekt „Translate NAMSE“, in dessen Rahmen über die Grundversorgung hinaus innovative Leistungen, vor allem strukturierte lokale und überregionale Fallkonferenzen und IT-gestützte Kommunikationswege zwischen den Versorgern etabliert und erprobt werden, mit dem Ziel, diese Strukturen in die Regelversorgung zu übernehmen. An diesem Projekt sind unter anderem die Kliniken für Neurologie der Universitätsklinika Tübingen, Bonn und Lübeck beteiligt. Auch werden vielerorts Verhandlungen mit den Kassen zur Bewilligung von Sonderpauschalen für Patienten mit seltenen Erkrankungen geführt, die den Stand von Spezialsprechstunden verbessern und perspektivisch nötige Freiräume für zeitintensive Prozesse ermöglichen werden.

Es gibt also Hoffnung, vor allem auch, weil die neuen Strukturmaßnahmen und sich abzeichnenden Erleichterungen einen Rahmen setzen zur Realisierung von Grundbedürfnissen und -erfordernissen in der Medizin: Diskurs, Diskussion, Beratung. Viele seltene neurologische Erkrankungen betreffen verschiedene Organsysteme, manifestieren sich im Kindes- oder Jugendalter und bestehen im Erwachsenenalter fort, stehen also exemplarisch für Erkrankungen, die sich nicht an Fächer- oder Altersgrenzen halten. Bei aller notwendigen Subspezialisierung wird eine Atomisierung umfassendes Verständnis dieser Erkrankungen vereiteln und Betroffenen und deren Familien nicht gerecht werden. Die Strukturen der ZSEs, der ERNs und weiterer Netzwerke laden, unterfüttert und flankiert von neuen IT-Lösungen (z. B. webbasierte Konsultationen, Clouds), zu fächerund altersübergreifendem Austausch ein.
Ausgehend von diesem Leitgedanken ist im November 2017 auch die Deutsche Akademie für Seltene Neurologische Erkrankungen (DASNE) gegründet worden. Sie wird in diesem Heft in einem Beitrag von Ludger Schöls, Holm Graessner und Alexander Münchau vorgestellt. Die DASNE versteht sich als interdisziplinäres altersübergreifendes Expertise-Netzwerk der Neurofächer, das in Ergänzung zur Nutzung telemedizinischer, webbasierter Strukturen dem persönlichen Gespräch und Austausch eines Experten-Panels aus Neurologie, Neuropädiatrie, Neuropsychiatrie, (Neuro-) Genetik und Neuroradiologie während eines jährlich auf der Wartburg in Eisenach stattfindenden Symposiums besonderen Raum gibt.

Weitere Artikel geben eine Übersicht über Themenfelder oder Syndrome, die beispielhaft für seltene Erkrankungen in den Neurofächern stehen, ohne dass an dieser Stelle ein Anspruch auf Vollständigkeit erhoben werden soll oder kann. Die jeweiligen Artikel, verfasst von Mitgliedern des DASNE-Experten-Panels, beleuchten besondere Charakteristika oder Entwicklungen bei definierten Erkrankungsgruppen, die für seltene Erkrankungen typisch sind. Josua Kegele, Sara von Spiczak, Markus Wolff und Holger Lerche widmen sich seltenen Epilepsien und paroxysmalen Störungen und zeigen auf, wie sich aus pathophysiologischen Erkenntnissen neue, teilweise hoch effektive Therapien ableiten lassen. Andres Ceballos-Baumann untermauert mit seinem Beitrag „Seltene Parkinson-Syndrome“, dass die Gruppe der häufigen Parkinson-Syndrome aus einer Vielzahl seltener Syndrome oder Erkrankungen besteht, die jeweils einer speziellen Evaluation und Behandlung bedürfen. Genetisch determinierte Dystonien werden von Norbert Brüggemann, Katja Lohmann, Sebastian Paus, Ebba Lohmann, Thomas Gasser, Christos Ganos und Christine Klein behandelt als Beispiel einer Gruppe von Syndromen, bei denen neurogenetische Forschungsansätze zu einer ganz erheblichen Vertiefung des Verständnisses geführt haben, das Eingang gefunden hat in eine neue Klassifikation und bei der Therapieplanung incl. der tiefen Hirnstimulation Orientierung zu geben beginnt. Das rasch wachsende komplexe 


\section{DASNE}

Spektrum der seltenen neuroimmunologischen Syndromen mit Autoantikörpern gegen ZNS-Antigene steht im Fokus des Artikels von Bettina Balint, Frank Leypold und Holger Lerche. Es handelt sich überwiegend um neuropsychiatrische Störungen mit teils sehr charakteristischer Phänomenologie, bei denen frühzeitige Diagnose und prompte Einleitung immunmodulierender Therapien prognostisch entscheidend sind. Birgit Assmann, Knut Brockmann, Eva Bültmann, Ingeborg Krägeloh-Mann und Thomas Opladen geben einen Überblick über die klinisch-diagnostisch herausfordernde Gruppe der Leukodystrophien und Neurotransmitterstörungen als neuropädiatrische Erkrankungen par excellence, die sich allerdings auch im
Erwachsenenalter manifestieren bzw. bei Betroffenen im Erwachsenenalter fortbestehen können und somit beispielhaft auch die Notwendigkeit einer engen Zusammenarbeit zwischen Neuropädiatern und Neurologen dokumentieren.

Wir wünschen Ihnen eine anregende und gewinnbringende Lektüre!

\section{Alexander Münchau, Lübeck}

\section{Literatur}

1. Romberg MH. Lehrbuch der Nervenkrankheiten des Menschen. Berlin: Duncker 1840.

2. Wehkamp KH, Naegler H. The commercialization of patient-related decision-making in hospitals - a qualitative study of the perceptions of doctors and chief executive officers. Dtsch Arztebl Int 2017; 114: 797-804.

3. Köbberling J. Economic pressure in hospitals profound disagreement over priorities between doctors and managers. Dtsch Arztebl Int 2017; 114: 795-6.

4. Schumm-Draeger PM, Kapitza T, Mann K, Fölsch U, Müller-Wieland D. Ökonomisierung in der Medizin. Rückhalt für ärztliches Handeln. Dtsch Arztebl Int 2017; 114: A2338-2340.

5. Schöls L, Gasser T, Krägeloh-Mann I, Graessner H, Klockgether T, Münchau A. Zentren für seltene neurologische Erkrankungen. Akt Neurol 2018; im Druck.

6. Graessner H, Schäfer F, Scarpa M et al. European Reference Networks: Consequences for healthcare in Germany. Bundesgesundheitsblatt Gesundheitsforschung Gesundheitsschutz 2017; 60: 537-541. 